\title{
Impact of Child and Informant Gender on Parent and Teacher Ratings of Attention-Deficit/Hyperactivity Disorder
}

By: Arthur D. Anastopoulos, Kaicee K. Beal, Rachel J. Reid, Thomas J. Power, Robert Reid, George J. DuPaul

Anastopoulos, A.D., Beal, K.K., Reid, R.J., Reid, R., Power, T.J., \& DuPaul, G.J. (2018). Impact of child and informant gender on parent and teacher ratings of attention-deficit/hyperactivity disorder. Psychological Assessment, 30(10), 1390-1394. http://dx.doi.org/10.1037/pas0000627.

\section{CAmerican Psychological Association, 2018. This paper is not the copy of record and may not exactly replicate the authoritative document published in the APA journal. Please do not copy or cite without author's permission. The final article is available, upon publication, at: http://dx.doi.org/10.1037/pas0000627}

\begin{abstract}
:
Attention-deficit/hyperactivity disorder (ADHD) rating scales typically provide normative tables arranged according to child age, child gender, and type of informant, which facilitates addressing the fifth edition of the Diagnostic and Statistical Manual of Mental Health Disorders developmental deviance requirement for diagnosing ADHD. Missing, however, is any consideration of the gender of the informant. The purpose of this paper was to conduct an exploratory examination of informant gender via secondary analyses of a large data set used to standardize the ADHD Rating Scale-5. Two (informant gender) by two (child gender) ANOVAs were conducted separately for parents and teachers using inattention (IN) symptoms, hyperactive-impulsive (HI) symptoms, the total impairment score related to IN, and the total impairment score for $\mathrm{HI}$ as dependent variables. Results showed that female parents rated male children significantly higher on both IN symptoms and impairment related to IN than did male parents. Female teacher ratings were also significantly higher than male teacher ratings for male children in terms of HI symptoms and with respect to impairment ratings related to both HI and IN. A significantly higher percentage of female parents (7.7\%) identified male children as being at risk for ADHD relative to male parents (4.1\%). This same pattern emerged for female teachers $(11.9 \%)$ versus male teachers $(5.3 \%)$. Such results suggest that informant gender may play a clinically meaningful role in assessing ADHD in children and adolescents, which is consistent with the developmental literature addressing gender stereotypes in children. Future research is needed to determine whether similar informant gender differences exist in other rating scale measures of ADHD.
\end{abstract}

Public Significance Statement 
Our study found that when parents and teachers rate attention-deficit/hyperactivity disorder (ADHD) behaviors in children, boys are nearly twice as likely to be identified as at risk for ADHD when rated by females versus males. Although preliminary, this finding suggests that it may be important for researchers and clinicians to take informant gender into account when assessing ADHD in children.

Keywords: attention-deficit/hyperactivity disorder | rating scales | children | informant | gender

\section{Article:}

Attention-deficit/hyperactivity disorder (ADHD) is a developmental disorder that arises in childhood, persists across the life span, and causes significant impairment in daily functioning (American Psychiatric Association, 2013). Approximately 6.5\% of the child and adolescent population exhibits ADHD (Polanczyk, Willcutt, Salum, Kieling, \& Rohde, 2014). For reasons that are not entirely clear, males are identified with ADHD at substantially higher rates than females (Barkley, 2015). Although there is a great deal of variability in the way that ADHD is evaluated, it is generally acknowledged that a multimethod, multiinformant approach is optimal for assessing ADHD and its co-occurring features and functional impairments (Anastopoulos \& Shelton, 2001). Rating scales are an indispensable component of the multimethod, multiinformant approach for assessing ADHD, and a large number and variety of narrow-band and broad-band measures have been developed for this purpose (Barkley, 2015).

As the name implies, narrow-band ADHD rating scales focus on the primary symptoms of this disorder and therefore are useful in deriving ADHD symptom frequency counts. Narrow-band ADHD measures for use with children and adolescents include the ADHD Rating Scale-5 (ADHD RS-5; DuPaul, Power, Anastopoulos, \& Reid, 2016), the Conners third edition (Conners, 2008a), the Swanson Nolan and Pelham Teacher and Parent Rating Scale (SNAP)-IV (Swanson, 1992), and the Vanderbilt ADHD Rating Scales (Wolraich, Feurer, Hannah, Baumgaertel, \& Pinnock, 1998). The ADHD RS-5 was developed in accordance with the fifth edition of the Diagnostic and Statistical Manual of Mental Health Disorders (DSM-5; American Psychiatric Association, 2013). Although the Conners third edition and the Vanderbilt were originally published before these newer diagnostic criteria were released, both have recently been updated to be compatible with DSM-5.

Broad-band rating scales address a wide range of child behaviors. In the context of ADHD assessment, they may be particularly useful in screening for conditions that co-occur with ADHD or that provide a better explanation for the presenting concerns. Commonly used broad-band rating scales include the Achenbach System of Empirically Based Assessment (Achenbach, 2009), the Behavior Assessment System for Children Third Edition (Reynolds \& Kamphaus, 2015), and the Conners Comprehensive Behavior Rating Scales (Conners, 2008b). 
Each of these measures has been published or updated since the release of the DSM-5. Each includes parent-, teacher-, and self-rated versions and provides adequate coverage of internalizing, externalizing, and social-developmental domains.

In addition to their flexibility, low cost, and convenience, many ADHD rating scales are standardized on large samples and provide normative tables that are arranged according to a child's age and gender and type of informant (i.e., parent or teacher). The availability of such normative tables makes rating scales especially useful in addressing the DSM-5 requirement for developmental deviance - that is, in determining whether the severity of reported ADHD symptoms is significantly deviant from developmental expectations for children of the same age and gender. Missing, however, from the previously mentioned rating scales is any consideration of the gender of the informant. The reasons for this omission are not entirely clear. This may stem from an underlying assumption that adult males and females do not differ in their perceptions of child behavior. Or it may simply be a practical matter, based on the belief that adult females are more likely to provide information about a child's behavior in home and school settings because they are generally more accessible. In either case, to the extent that informant gender differences may exist, such differences could have important implications for diagnostic evaluations and the assessment of treatment outcome.

As a first step in addressing this situation, the purpose of this paper was to conduct an exploratory examination of these informant gender issues via secondary analyses of a large data set used to standardize the recently published ADHD RS-5 (DuPaul et al., 2016). In addition to traditional group-based statistical analyses, the data were examined at an individual level to explore the potential clinical impact that informant gender may have on ADHD diagnostic rates.

\section{Method}

The ADHD RS-5 (DuPaul et al., 2016) contains items addressing not only ADHD symptoms but also impairment related to those symptoms, which can be completed by parents and teachers for children 5-17 years of age. With the permission of the American Psychiatric Association, 18 ADHD items were created based on the wording used to describe those items in the DSM-5. Consistent with the DSM-5 and with the results of multiple factor analytic studies (DuPaul et al., 2016; Molina, Smith, \& Pelham, 2001), the 18 items are presented in the context of a two-factor structure, beginning with the nine inattention (IN) symptoms followed by the nine hyperactive-impulsive (HI) symptoms. Parents and teachers rate each of these items on a 4-point Likert frequency scale that can be scored 0 (never or rarely), 1 (sometimes), 2 (often), or 3 (very often). IN and HI total symptom severity scores are derived by summing the actual ratings for the nine IN items (range $0-27)$ and the nine $\mathrm{HI}$ items $(0-27)$, respectively. To facilitate mapping onto DSM-5 criteria, these same items can be scored categorically to generate

$\mathrm{IN}$ and HI symptom counts. The symptom count for IN is determined by summing the number of $\mathrm{IN}$ items receiving ratings of 2 (often) or 3 (very often). The symptom count for $\mathrm{HI}$ is calculated 
in a similar fashion. Thus, for both IN and HI, symptom counts range from 0 to 9 in accordance with DSM-5 criteria.

In addition to the descriptions of the nine IN symptoms and the nine HI symptoms, the ADHD RS-5 contains six items assessing functional impairments that are commonly reported for children with ADHD and thus found in other impairment rating scales (Fabiano et al., 2006). These six items address relationships with significant adults, peer relationships, academic functioning, behavioral functioning, homework, and self-esteem. After completing their responses to the nine IN symptoms, informants are asked, "How much do the nine behaviors in the previous question cause problems for your child." Informants than rate each of the six impairment items using a 4-point scale (no problem, minor problem, moderate problem, severe problem). In a similar fashion, informants then rate these same six impairment items after completing their responses to the nine HI symptoms. Although each impairment item receives a rating for both IN and HI symptoms, only the higher of these two ratings is selected for further analysis. Such a procedure allows for the possibility that certain types of impairment may be related to IN symptoms, whereas other types of impairment may stem from HI symptoms. From this final group of six, the number of impairment items with ratings of moderate or severe is then calculated to yield an overall single impairment score ranging from 0 to 6 . Overall impairment scores that are greater than or equal to 3 for parents or greater than or equal to 5 for teachers are considered clinically significant (Power et al., 2017).

The parent sample used to standardize the ADHD RS-5 was recruited from all regions of the United States and included households from both metropolitan (86.4\%) and nonmetropolitan $(13.6 \%)$ locations. All parent respondents were recruited through a national research firm, GfK. Specifically, the GfK Knowledge Panel was used to generate a sample of children and adolescents representative of the U.S. population in terms of race, ethnicity, geographic region, and family income. Parents were selected using address-based sampling that allows probability-based sampling of addresses from the U.S. Postal Service's Delivery Sequence File. Individuals residing at randomly sampled home addresses were invited to join the GfK Knowledge Panel through a series of mailings in both English and Spanish. Selected parents indicated their willingness to join the panel either by returning a completed acceptance form in a postage-paid envelope, calling a toll-free hotline, speaking to a bilingual recruitment agent, or accessing a dedicated recruitment website. A total of 4,219 individuals was initially contacted to participate and 2,708 (64.2\%) completed parent ratings. To create a sample that closely approximated U.S. Census reported rates of targeted child demographics (i.e., age, gender, race, ethnicity, family income, geographic region), 719 ratings were removed, resulting in a final sample of 2,079 parent ratings that were included in the analyses (DuPaul et al., 2016).

Included in this sample were 1,131 female and 948 male parents who completed ADHD RS-5 symptom and impairment ratings for one child in their family. For families with more than one child, the rated child was selected randomly by the researchers. Parents and guardians were predominantly Caucasian (64.1\%), 20 -77 years of age (M 41.6; SD 8.2), married (79.7\%), high 
school/college educated (89.9\%), and employed (72.3\%). Median household income fell between $\$ 60,000$ and $\$ 74,999$. The parent-rated children included 1,037 males and 1,042 females ranging in age from 5 to 17 years (M 10.7; SD 3.75); approximately $23.4 \%$ of these children were Hispanic, 53.9\% were Caucasian, 13.1\% were African American (53.9\%), 3.9\% were multiracial, and $5.9 \%$ were from other racial groups.

A portion of the teacher data was also collected via the GfK Knowledge Panel. An initial pool of 1,509 teachers was invited to participate and 1,019 (67.5\%) completed ratings. Following the same U.S. Census guidelines that were used for the parent sample, it was necessary to remove a high percentage of teacher ratings, leaving only 474 teacher ratings available for further analyses. Because this number of GfK Knowledge Panel teachers who completed ratings fell well short of the targeted enrollment goal $(1,000)$, it became necessary to recruit additional teachers via a second national research firm, e-Rewards Market Research. The e-Reward panelists are selected based on having a relationship with a business (e.g., Pizza Hut, Macy's). A double opt-in is required, such that panelists must reply to the initial e-mail invitation and then to a follow-up confirmation e-mail. Of the 12,610 teachers who were invited to participate in this manner, 1,399 (11.1\%) completed ratings, and 596 (42.6\%) were retained for inclusion in the analyses based on a consideration of the same targeted child demographics noted above.

The final sample included 1,070 teachers ( 766 female, 304 male) who completed ADHD symptom and impairment ratings for two students (one male, one female) randomly selected from their class rosters by the researchers. Teachers from both general (83.3\%) and special education (16.4\%) classroom settings participated. Teachers were predominantly non-Hispanic Caucasian (87.3\%), with a mean of 17.9 years of teaching experience (SD 10.7). The students (N 2,140; 1,070 males, 1,070 females) rated by the teachers were in kindergarten through 12th grade and ranged in age from 5 to 17 years (M 11.53; SD 3.54). In terms of race and ethnicity, approximately $24.0 \%$ of these children came from Hispanic backgrounds, $54.8 \%$ were Caucasian, $12.7 \%$ identified as African American (53.9\%), 1.5\% were multiracial, and 7.0\% were from other racial groups.

Parents and teachers completed their ratings through a web-based survey that took approximately 5-10 min. Respondents received small stipends (less than \$5) for their participation. All ratings were collected during April and May of 2014.

\section{Results and Discussion}

ANOVAs were conducted separately for parents and teachers using informant gender and child gender as the independent variables. IN symptom count, HI symptom count, the total impairment score related to IN, and the total impairment score for HI were analyzed separately as the dependent variables.

Obtained 2 (informant gender) 2 (child gender) ANOVA results revealed statistically significant interactions within both informant data sets (see Table 1). More specifically, whereas 
there were no significant parental gender differences in the ratings of female children, female parents rated male children higher on both IN symptom counts and impairment related to IN than did male parents. Partial eta squared values were .002 for both IN symptom counts and impairment related to IN, suggesting very small effect sizes. Although not reaching a level of statistical significance, trends emerged showing this same parent gender difference with respect to HI symptom counts and impairment related to HI.

In line with the parent ratings, there were no significant teacher gender differences in the ratings of female children. Female teacher ratings, however, were significantly higher than male teacher ratings for male children in terms of $\mathrm{HI}$ symptom counts and with respect to impairment ratings related to both HI and IN. Partial eta squared values were .002 for HI symptom count, .002 for impairment related to HI, and .001 for impairment related to IN, again suggesting very small effect sizes. Also evident was a trend showing this same teacher gender difference with respect to IN symptom counts.

To assess the potential clinical impact of these ANOVA findings, children were first classified as being at risk for ADHD if they met two of the five criteria for ADHD listed in the DSM-5. Specifically, children were considered at risk for ADHD if they were rated as displaying six or more symptoms of either IN or HI, and impairment ratings for either IN or HI that fell in a developmentally deviant range based on the ADHD RS-5 norms. Such a classification approach is consistent with recent findings indicating that joint consideration of symptom counts and impairment yields ADHD prevalence estimates most consistent with prior epidemiological findings (DuPaul, Reid, Anastopoulos, \& Power, 2014).

For both parents and teachers, 2 analyses showed that there were no significant differences in the percentages of female and male informants who identified female children as being at risk for ADHD (see Table 2). In contrast, a significantly higher percentage of female parents $(7.7 \%)$ identified male children as being at risk for ADHD relative to male parents (4.1\%). This same pattern emerged for female teachers (11.9\%) versus male teachers (5.3\%). Phi values were .08 and .10 for the parent and teacher findings, respectively, suggesting small effect sizes.

Taken together, the obtained results suggest that informant gender may play a clinically meaningful role in determining both the severity and classification of ADHD in children and adolescents. To the best of our knowledge, such a finding has not been previously reported. Replication studies should therefore be undertaken to determine whether a similar pattern of informant gender differences occurs in other well-established rating scale measures of ADHD. Likewise, it would seem prudent to consider the possibility that similar informant gender differences may exist among rating scales that measure constructs other than ADHD (e.g., conduct problems, emotional functioning, social-adaptive functioning, and self-esteem in children).

Although the obtained effect sizes in this study were small in magnitude, it is critically important to keep in mind the clinical implications of the categorical analyses of at-risk ADHD 
rates. This is because being diagnosed with ADHD sets in motion a multitude of changes in a child's life, including changes in self-perception, changes in others' perceptions, and the likelihood of receiving stimulant medication and other forms of treatment. The need for accurate diagnosis is therefore extremely important (Anastopoulos \& Shelton, 2001), and such a diagnosis should not be dependent on the gender of the parent or teacher completing ratings.

The fact that adult males and females differed in their ratings of ADHD symptoms and related impairments is consistent with findings in the developmental literature addressing gender stereotypes in children. In one study, for example, gender stereotypes held by mothers and fathers interacted with child gender in predicting parental beliefs about their child's academic abilities (Tiedemann, 2000). More generally, research has shown that child gender affects parents' causal attributions for their child's performance in a variety of activities and that these parental biases influence children's self-perceptions and activity choices (Eccles, Jacobs, \& Harold, 1990).

Because these findings were drawn from a community sample, it will be important to examine this issue in a follow-up study with a sample that includes clinic-referred children with a well-established diagnosis of ADHD. Another limitation impacting the generalizability of these findings stems from a consideration of the less than optimal rates at which parents and teachers responded to the invitations to participate in the study. Although findings from the current study suggest that informant gender may impact ratings of ADHD symptoms in children, such an interpretation may be an oversimplification of a clinical phenomenon that is much more complex in nature. Other child factors, such as age, ADHD medication status, or co-occurring behavioral or emotional problems, might reasonably come into play. Being depressed, having ADHD, or perhaps living with a family member who has ADHD are just a few of the many possible informant characteristics that could also impact parent and teacher ratings of ADHD symptoms in children. Contextual factors, such as family size and length of teaching experience, would also need to be considered as possible influences on parent and teacher ratings, respectively.

Bearing these limitations in mind, findings from the current study nevertheless raise the possibility that informant gender may be among the many factors working together to impact parent and teacher ratings of ADHD symptoms in children. Although not yet addressed empirically, similar informant gender differences may also influence parent and teacher ratings of various other child characteristics (e.g., anxiety, peer relations). Assuming such an informant gender bias does indeed exist, it may ultimately be necessary for future rating scales to provide normative tables in which gender is taken into consideration within each type of informant (i.e., parent or teacher). In the meantime, researchers and clinicians alike would be well advised to take informant gender into account by routinely including female and male informants in their assessments of children at risk for ADHD. 
Table 1. Summary of ANOVA Results for Informant Gender by Child Gender Interactions

\begin{tabular}{llcc}
\hline Informant & \multicolumn{1}{c}{ Variable } & $F(1)$ & $p$ \\
\hline Parent & IN symptom count & 4.16 & .042 \\
& HI symptom count & 2.57 & .109 \\
& Impairment related to IN & 4.33 & .038 \\
\multirow{4}{*}{ Teacher } & Impairment related to HI & 3.28 & .070 \\
& IN symptom count & 2.56 & .109 \\
& HI symptom count & 5.22 & .022 \\
& Impairment related to IN & 2.72 & .010 \\
& Impairment related to HI & 4.70 & .030 \\
\hline
\end{tabular}

Note. $\mathrm{IN}=$ inattention; $\mathrm{HI}=$ hyperactive-implusive.

Table 2. Summary of At-Risk ADHD Classification Rates by Informant and Child Gender

\begin{tabular}{cllcrr}
\hline $\begin{array}{c}\text { Informant type } \\
\text { gender }\end{array}$ & $\begin{array}{c}\text { Informant } \\
\text { gender }\end{array}$ & Child ADHD & At-risk, \% & $\chi^{2}(1)$ & $p$ \\
\hline \multirow{2}{*}{ Parent } & Male & Male & 4.1 & 5.85 & .02 \\
& Female & Male & 7.7 & & \\
& Male & Female & 3.1 & .07 & .79 \\
& Female & Female & 3.4 & & \\
Teacher & Male & Male & 5.3 & 10.59 & .001 \\
& Female & Male & 11.9 & & \\
& Male & Female & 4.3 & .41 & .52 \\
& Female & Female & 5.2 & & \\
\hline
\end{tabular}

\section{References}

Achenbach, T. M. (2009). The Achenbach System of Empirically Based Assessment (AESBA): Development, findings, theory, and applications. Burlington, VT: University of Vermont Research Center for Children, Youth, and Families.

American Psychiatric Association. (2013). Diagnostic and statistical manual of mental disorders (5th ed.). Washington, DC: American Psychiatric Association.

Anastopoulos, A. D., \& Shelton, T. L. (2001). Assessing attention-deficit/ hyperactivity disorder. New York, NY: Kluwer Academic/Plenum Press.

Barkley, R. A. (2015). Attention-deficit/hyperactivity disorder: A handbook for diagnosis and treatment (4th ed.). New York, NY: Guilford Press.

Conners, C. K. (2008a). Conners (3rd ed.). Toronto, ON, Canada: MultiHealth Systems.

Conners, C. K. (2008b). Conners Comprehensive Behavior Rating Scales. North Tonawanda, NY: Multi-Health Systems.

DuPaul, G. J., Power, T. J., Anastopoulos, A. D., \& Reid, R. (2016). ADHD Rating Scale-5 for Children and Adolescents: Checklists, norms, and clinical interpretation. New York: Guilford Press.

DuPaul, G. J., Reid, R., Anastopoulos, A. D., Lambert, M. C., Watkins, M. W., \& Power, T. J. (2016). Parent and teacher ratings of attention deficit/hyperactivity disorder symptoms: 
Factor structure and normative data. Psychological Assessment, 28, 214 -225. http://dx.doi.org/10.1037/ pas0000166

DuPaul, G. J., Reid, R., Anastopoulos, A. D., \& Power, T. J. (2014). Assessing ADHD symptomatic behaviors and functional impairment in school settings: Impact of student and teacher characteristics. School Psychology Quarterly, 29, 409 - 421. http://dx.doi.org/10.1037/spq0000095

Eccles, J. S., Jacobs, J. E., \& Harold, R. D. (1990). Gender role stereotypes, expectancy effects, and parents' socialization of gender differences. Journal of Social Issues, 46, 183-201. http://dx.doi.org/10.1111/j.1540- 4560.1990.tb01929.x

Fabiano, G. A., Pelham, W. E., Jr., Waschbusch, D. A., Gnagy, E. M., Lahey, B. B., Chronis, A. M.,... Burrows-Maclean, L. (2006). A practical measure of impairment: Psychometric properties of the impairment rating scale in samples of children with attention deficit hyperactivity disorder and two school-based samples. Journal of Clinical Child and Adolescent Psychology, 35, 369 -385. http://dx.doi.org/10.1207/s15374424jccp3503_3

Molina, B. S. G., Smith, B. H., \& Pelham, W. E. (2001). Factor structure and criterion validity of secondary school teacher ratings of ADHD and ODD. Journal of Abnormal Child Psychology, 29, 71- 82. http://dx.doi .org/10.1023/A:1005203629968

Polanczyk, G. V., Willcutt, E. G., Salum, G. A., Kieling, C., \& Rohde, L. A. (2014). ADHD prevalence estimates across three decades: An updated systematic review and meta-regression analysis. International Journal of Epidemiology, 43, $434-442$. http://dx.doi.org/10.1093/ije/ dyt261

Power, T. J., Watkins, M. W., Anastopoulos, A. D., Reid, R., Lambert, M. C., \& DuPaul, G. J. (2017). Multi-informant assessment of ADHD symptom-related impairments among children and adolescents. Journal of Clinical Child and Adolescent Psychology, 46, 661674. http://dx.doi .org/10.1080/15374416.2015.1079781

Reynolds, C. R., \& Kamphaus, R. W. (2015). Behavior Assessment System for Children (3rd ed.). Bloomington, MN: Psych Corp. Swanson, J. M. (1992). School-based assessments and interventions for ADD students. Irvine, CA: KC Publishing.

Tiedemann, J. (2000). Parents' gender stereotypes and teachers' beliefs as predictors of children's concept of their mathematical ability in elementary school. Journal of Educational Psychology, 92, 144 -151. http://dx .doi.org/10.1037/0022-0663.92.1.144

Wolraich, M. L., Feurer, I. D., Hannah, J. N., Baumgaertel, A., \& Pinnock, T. Y. (1998). Obtaining systematic teacher reports of disruptive behavior disorders utilizing DSM-IV. Journal of Abnormal Child Psychology, 26, 141-152. http://dx.doi.org/10.1023/A:1022673906401

This article was published Online First June 25, 2018.

Arthur D. Anastopoulos, Kaicee K. Beal, and Rachel J. Reid, Department of Human Development and Family Studies, University of North Carolina at Greensboro; Robert Reid, 
Department of Special Education and Communication Disorders, University of

Nebraska-Lincoln; Thomas J. Power, Department of Child and Adolescent Psychiatry and Behavioral Sciences, Children's Hospital of Philadelphia, Philadelphia, Pennsylvania; George J. DuPaul, Department of Education and Human Services, Lehigh University.

George J. DuPaul, Thomas J. Power, Arthur D. Anastopoulos, and Robert Reid have a financial interest in the ADHD Rating Scale-5, which was used in this study to assess parent and teacher ratings of ADHD symptoms and impairment.

Correspondence concerning this article should be addressed to Arthur D. Anastopoulos, Department of Human Development and Family Studies, University of North Carolina at Greensboro, Greensboro, NC 27402. E-mail: ada@uncg.edu 